\title{
Excitonic coupling directly revealed on single chains of polyfluorene by combined force spectroscopy and fluorescence microscopy
}

\section{Tomonori Nakamura}

Tokyo Institute of Technology

\section{Shun Omagari}

Tokyo Institute of Technology

Xiaobin Liang

Tokyo Institute of Technology

Ken Nakajima

Tokyo Institute of Technology

Martin Vacha ( $\nabla$ vacha.m.aa@m.titech.ac.jp )

Tokyo Institute of Technology https://orcid.org/0000-0002-5729-9774

\section{Article}

Keywords: molecular aggregates, excitonic coupling, molecular photophysics, energy conversion mechanism

Posted Date: August 26th, 2021

DOI: https://doi.org/10.21203/rs.3.rs-820297/v1

License: (c) (i) This work is licensed under a Creative Commons Attribution 4.0 International License. Read Full License 
Excitonic coupling directly revealed on single chains of polyfluorene by combined force spectroscopy and fluorescence microscopy

Tomonori Nakamura ${ }^{1}$, Shun Omagari ${ }^{1}$, Xiaobin Liang ${ }^{2}$, Ken Nakajima ${ }^{2}$ and Martin Vacha $^{1 *}$

${ }^{1}$ Department of Materials Science and Engineering, School of Materials and Chemical Engineering, Tokyo Institute of Technology, Ookayama 2-12-1-S8-44, Meguro-ku, Tokyo 152-8552, Japan.

${ }^{2}$ Department of Chemical Science and Engineering, School of Materials and Chemical Engineering, Tokyo Institute of Technology, Ookayama 2-12-1, Meguro-ku, Tokyo $152-$ 8552, Japan. 


\section{Abstract}

Molecular aggregates were discovered in 1930's, yet, the forces and excitonic coupling energy associated with the aggregate formation have not been detected so far. We directly measure such force and energy on single chains of the conjugated polymer polyfluorene using atomic force and fluorescence microscopes. The polyfluorene chain is attached on either side to a substrate and an AFM tip, respectively, and mechanically stretched under intense laser irradiation. The force-extension curves show force peaks that are attributed to gradual unfolding of the chain. Upon the irradiation, neighboring conjugated segments interact via excitonic coupling when in contact and experience an attractive force which is detected by the AFM. Analysis of the force curves provides excitonic coupling energy which is of same order as theoretically calculated values for a face-to-face fluorene dimer, and in agreement with the energy obtained from single-chain fluorescence spectra. Apart from contributing an essential piece of knowledge in the field of molecular photophysics, the work demonstrates on molecular scale a novel energy conversion mechanism from light to mechanical energy which could be potentially used, e.g., as a driving mechanism for molecular motors. 
The exceptional photophysical properties of molecular aggregates continue to fascinate scientists since their discovery ${ }^{1,2}$. Upon mixing in solutions under specific conditions, certain organic dyes can form both $\mathrm{J}$ - and $\mathrm{H}$-aggregates, characterized by varying color and distinct spectral and photophysical features ${ }^{3-5}$. Over the decades, great effort has been spent on synthesis of novel dyes and utilization of the unique properties of molecular aggregates for functional materials ${ }^{6.7}$. The phenomenon is not restricted to aggregation of small-molecule dyes but has been also observed to cause some of the characteristic properties of light absorbing and emitting conjugated polymers ${ }^{8}$. The signature spectral features of molecular aggregates, the bathochromic (for J-aggregates) and hypsochromic (for H-aggregates) absorption shifts, have been successfully explained by the dye geometrical arrangement in Kasha's theory ${ }^{3}$. The theory itself has roots in the concept of molecular excitons $s^{9}$ and its application in molecular crystals ${ }^{10}$. The spectral shifts are explained due to splitting of the excited state of the aggregate caused by dipole-dipole interaction between transition dipole moments of neighboring molecules. The amount of the splitting, which provides the exciton coupling energy, depends on the distance between the molecules, their arrangement and on the magnitude of their transition dipoles. The arrangement of the molecules also determines the allowed or forbidden character of optical transitions into the split states, which in turn determines the $\mathrm{H}$ - or J- character of the aggregates. While the Kasha's theory is relatively simple, it has been successfully used to qualitatively describe experimental results on a range of molecular structures over the decades. Still, there has been so far no direct measurement of the energy associated with the exciton coupling, other than by spectroscopic means.

The dipole-dipole interaction between two neighboring molecules is an attractive one, leading to a strongly bound pair in the lower energy level of the split excited state. 
Separating such bound state under illumination therefore requires mechanical force that can be, in principle, provided and measured by atomic force microscopy (AFM). The feasibility of such approach has been shown theoretically for force spectroscopy on dimers of perylene and terylene derivatives which were estimated to require forces on the order of $\mathrm{pN}$ to tens of $\mathrm{pN}$ to break the excitonic bond ${ }^{11}$. Experimentally, force spectroscopy based on stretching of single macromolecular chains with an AFM cantilever has become an established technique that has been used to study conformational changes, nanorheology, mechanical dynamics and function of DNA, proteins as well as synthetic polymers ${ }^{12-18}$. Further, the single-chain force spectroscopy has been combined with optical microscopy to demonstrate an optomechanical cycle on photochromic dyes ${ }^{19}$ or to study and manipulate protein conformations ${ }^{20,21}$.

Synthetic conjugated polymer chains in compact conformations or in films often exhibit spectral properties that can be explained as a result of interactions between individual conjugated segments of the chain. Conjugated segments take a role of individual chromophores and interaction between their transition dipole moments gives rise to phenomena that are well known for aggregates of small molecules, including J- or $\mathrm{H}$-aggregation or excimer formation ${ }^{22-25}$. A good example is the conjugated polymer polyfluorene which is known for its outstanding optoelectronic properties including bright blue emission in solution ${ }^{26,27}$. Under specific conditions the blue emission can be accompanied by a green emission band, the origin of which has been a subject of longstanding debates ${ }^{28}$, but which has recently been shown, on single-molecule level, to arise from intra-chain aggregates ${ }^{29,30}$.

Here, we directly measure the excitonic coupling energy associated with molecular aggregates on single polyfluorene chains using a combination of force spectroscopy and 
fluorescence microscopy. The polyfluorene chain is mechanically stretched by an AFM cantilever from a self-folded conformation into completely stretched state, and the stretching is accompanied with intense laser irradiation close to the polyfluorene absorption peak. Under such conditions, the force curve of the chain unfolding shows small force peaks that are attributed to excitonic coupling of an intra-chain $\mathrm{H}$-aggregate.

\section{Single PFO chain stretching in dark}

Single chain force spectroscopy was performed with poly(9,9-dioctylfluorene) (PFO) terminated at both ends with amino groups. The amino groups react on either ends with epoxy groups on the surface of silane-functionalized quartz substrate and silicon cantilever to form covalent bonds (Fig. 1a). Immobilization of individual aminoterminated PFO chains on the functionalized surface was confirmed by single-chain fluorescence microscopy and spectroscopy (Supplementary Information, Supplementary Fig. 1 and 2). The force experiments are carried out in toluene using an AFM head placed on top of an inverted fluorescence microscope. Typical force spectra (force - extension curves) in Fig. 1b, c show single force peaks with a magnitude of several hundred pN, indicating that the amino-terminated PFO chains are correctly picked up by the functionalized AFM cantilever and extended to full length before the bond breakage. The force spectra are well fit with a modified freely jointed chain model (m-FJC) ${ }^{31}$ which accounts for the chain elasticity. The extension length at the bond breakage, the rupture length, corresponds to the length of a fully stretched polymer chain. The rupture lengths of 51 single PFO chains plotted in a histogram in Fig. 1d have an average value of 80.7 $\mathrm{nm}$, longer than $60 \mathrm{~nm}$ of the full length of the PFO chain as estimated from the molecular weight and quantum chemical size of a fluorene monomer. 


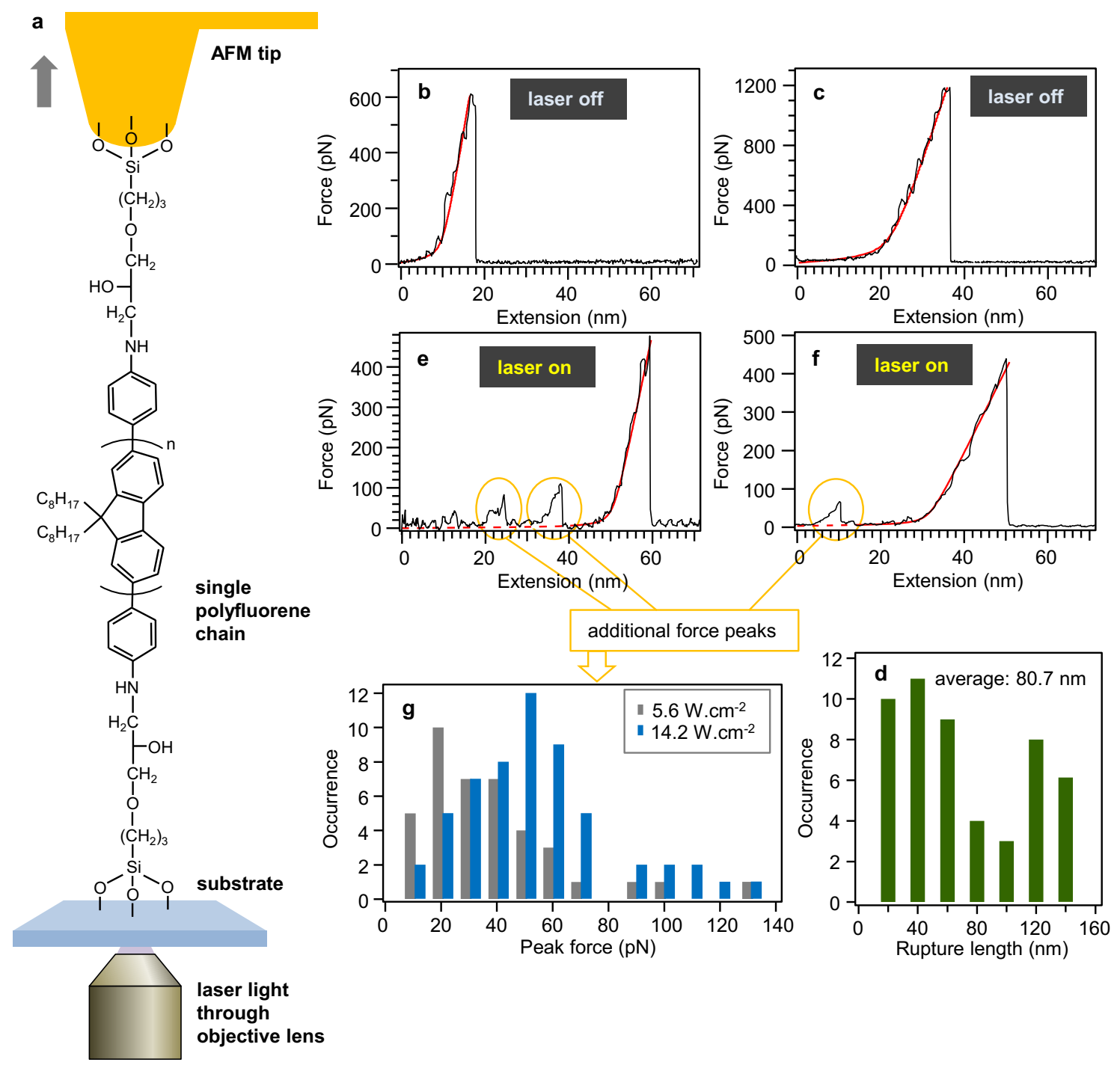

Fig. 1. Experimental force spectroscopy on single PFO chains under laser illumination. a, Chemical structure of the amino-terminated PFO and scheme of the force spectroscopy measurement. b, c, Force - extension curves of single PFO chains measured without the presence of light. The red lines are fits using the m-FJC model. d, Distribution of rupture lengths (lengths of fully stretched chains at bond breakage). e, f, Same as b, $\mathbf{c}$, measured under illumination of $375 \mathrm{~nm}$ laser with power of $14.2 \mathrm{~W} \cdot \mathrm{cm}^{-2}$; the fits involve the origin of the stretching curves. $\mathbf{g}$, Distribution of the peak values of the small force peaks appearing only under laser illumination, for two different laser powers as indicated in the legend. 
The larger average rupture length value indicates that longer polymer chains are more easily picked up by the AFM cantilever because the amine-terminated free end of the polymer chain diffuses in a hemisphere whose diameter corresponds to chain length, and a larger hemisphere increases the reaction probability.

\section{Single PFO chain stretching under illumination}

Typical force spectra in the presence of $14.2 \mathrm{~W} . \mathrm{cm}^{-2}$ of $375 \mathrm{~nm}$ laser illumination are shown in Fig. 1e, f. The main force peaks are similar to those obtained without the illumination, including good fits with the m-FJC model. The main difference is the appearance of additional small force peaks in the initial stages of the stretching. These small force peaks appear only under the laser illumination, as verified on 55 single PFO chains under illumination vs. 51 single chains stretched in dark. A histogram of the magnitude of the small force peaks in Fig. $1 \mathrm{~g}$ has an average value of $47 \pm 2 \mathrm{pN}$, an order of magnitude agreement with the theoretically predicted value for excitonic coupling in perylene dimers ${ }^{11}$. To further confirm the light-induced origin of the small force peaks, we performed the same experiment with reduced light intensity of $5.6 \mathrm{~W} . \mathrm{cm}^{-2}$. The average force value obtained from the histogram of 40 single chains (Fig. 1g) is $29 \pm 2$ $\mathrm{pN}$. While not decreasing proportionally with the light intensity, this results still verifies the light-induced nature of the phenomenon. The lack of proportionality is likely caused by the inability to detect small force peaks under noise reaching up to $10 \mathrm{pN}$.

\section{Analysis of the coupling energy}

The force spectroscopy results are further analyzed and interpreted in terms of excitonic coupling between conjugated segments of a folded PFO chain, as schematically shown in 
Fig. 2a. In the initial phases of stretching under illumination, two conjugated segments are coupled face-to-face in H-aggregate manner. This excitonic coupling is broken as the chain is lifted by stretching (Fig. 2b), resulting in the appearance of the small force peak (Fig. 2c). Integration of the force-extension curve provides energy associated with the excitonic coupling. Further, the extension length along the force peak is related to the coupling length (shown in Fig. 1b). Both quantities were analyzed on an ensemble of 55 single PFO chains and are shown in the histograms in Fig. 2d, e. Peak value of the coupling energy distribution is $0.54 \pm 0.08 \mathrm{eV}$. To evaluate the appropriateness of this number and to facilitate comparison with theoretical calculations or with spectroscopic data, this value has to be corrected so that it corresponds to coupling energy between two conjugated segments, one of which is in the excited state at all time. The laser light used in this work is a picosecond pulsed source. The average number of conjugated segments excited by one laser pulse can be estimated using the pulse energy and average absorption cross section to obtain 1.6 conjugated segments per pulse. The absorption can happen anywhere on the chain but the excitation energy is quickly funneled to the lowest energy site which is the aggregate. Further, the laser repetition rate of $80 \mathrm{MHz}$ results in a laser pulse arriving every $12.5 \mathrm{~ns}$. Excited state lifetime of the green band emission of PFO has two components, a shorter one of $1.4 \mathrm{~ns}$ which is spectrally centered around $500 \mathrm{~nm}$, and a longer one of $5.1 \mathrm{~ns}$ which spectrally corresponds to a region between 520 and $575 \mathrm{~nm}^{30}$. Considering the longer component as representing the weakly emissive H-aggregate, we use the $5.1 \mathrm{~ns}$ and calculate that the aggregate excited state is populated on average $41 \%$ of the time. 

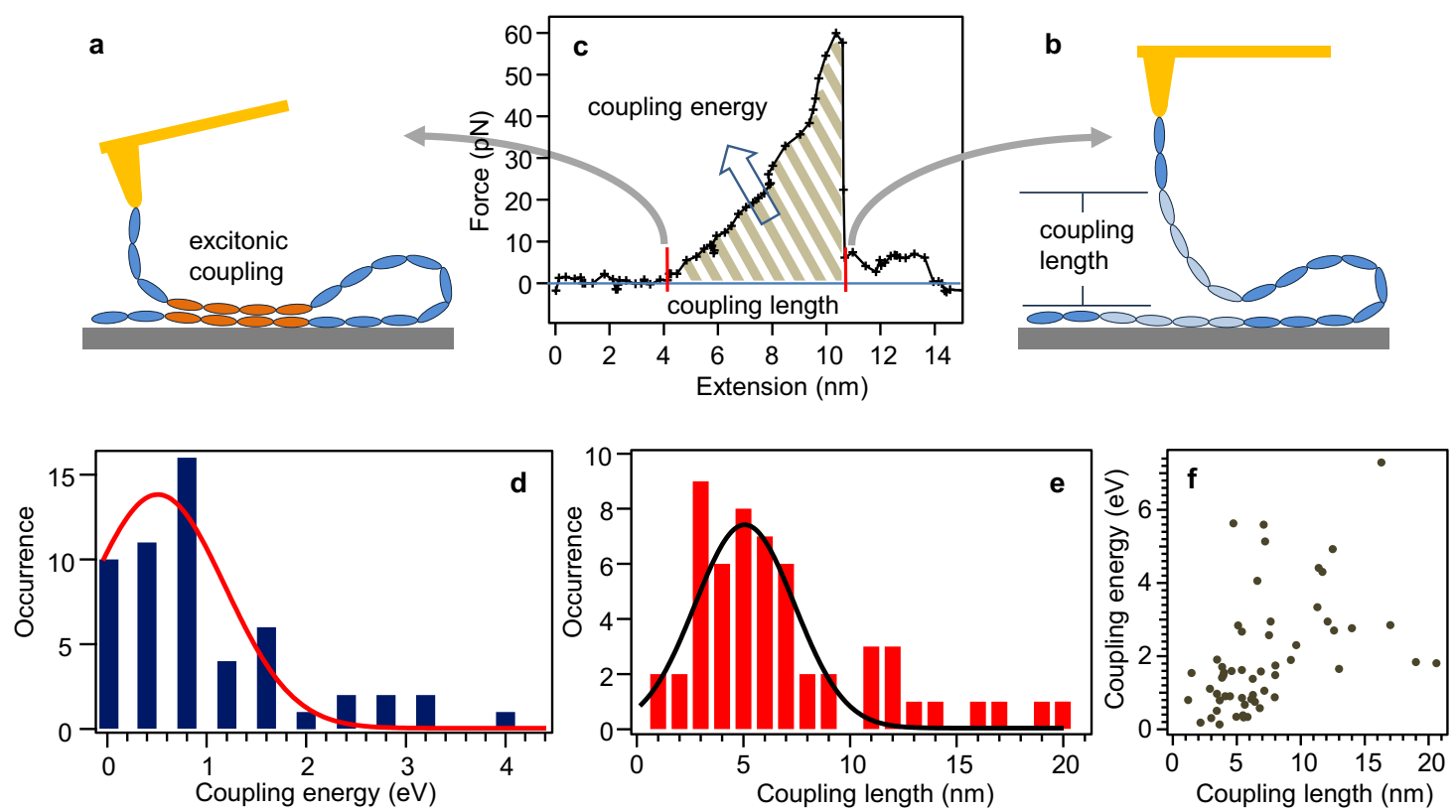

Fig. 2. Analysis of light-induced force peaks from force-extension curves. a, Schematic illustration of the initial phases of stretching of a folded PFO chain, with the part of the chain forming an aggregate highlighted in orange. b, Schematic illustration of breaking of the aggregate by the chain stretching, depicting the coupling length. c, Detail of the light-induced small force peak, showing schematically the coupling energy and length parameters. d, Distribution of the excitonic coupling energy obtained under illumination with 14.2 W. $\mathrm{cm}^{-2}$ from 55 single PFO chains. The red line is a Gaussian fit of the distribution. e, Distribution of coupling lengths from the same 55 PFO chains, with the black line is a Gaussian fit of the distribution. $\mathbf{f}$, Correlation between the distributions in $\mathbf{d}$ and $\mathbf{e}$.

Taking the two correction factors into account, we can estimate that the excitonic coupling energy of one conjugated segment is $(0.54 \mathrm{eV} / 1.6) / 0.41=0.82 \mathrm{eV}$. This value corresponds to the peak of the coupling energy distribution in the Fig. 2d. The distribution itself is broad with a long tail towards high energy values. Such distribution is caused 
mainly by the distribution of the PFO molecular weights (with the polydispersity index of 1.61) which results in larger absorption cross-sections and larger numbers of conjugated segments excited in the longer chains.

Distribution of the coupling lengths (as defined in the Fig. 2c) has a peak at $4.9 \pm$ $0.3 \mathrm{~nm}$. Considering the size of a fluorene monomer, this corresponds to about 6 monomer units. This is a very reasonable number since the conjugated segment length of glassyphase PFO is about 8 monomers $^{32}$, and the measured coupling length provides a lower limit of the actual length of the segment that took part in the aggregate, as seen in Fig. 2c. The measured coupling energy and coupling lengths are generally well correlated, as shown in Fig. 2f. Such correlation can be expected, since longer contact length should lead to higher energy. The deviations observed for several points in the longer lengths region are probably caused by geometry factors (distance or orientation) that result in weaker coupling.

\section{Comparison with quantum chemical calculations and single-chain fluorescence}

Feedback on the values obtained from the force spectroscopy can be provided by quantum chemical calculations and by spectroscopic data. The Fig. 3a shows the molecular aggregate energy scheme as introduced in the Kasha's model. Splitting of the $\mathrm{S}_{1}$ energy level provides the excitonic coupling energy which can be also obtained from a spectral shift between monomer and aggregate fluorescence bands. An example of a fluorescence spectrum of a single PFO chain measured in softened polystyrene matrix during solvent vapor annealing ${ }^{33}$ is shown in Fig. 3b. Under such conditions, the PFO spectrum shows dynamic changes between the monomer spectrum peaked around $420 \mathrm{~nm}$ and the green band between 530 and $580 \mathrm{~nm}$, due to the chain conformational dynamics ${ }^{33}$. 

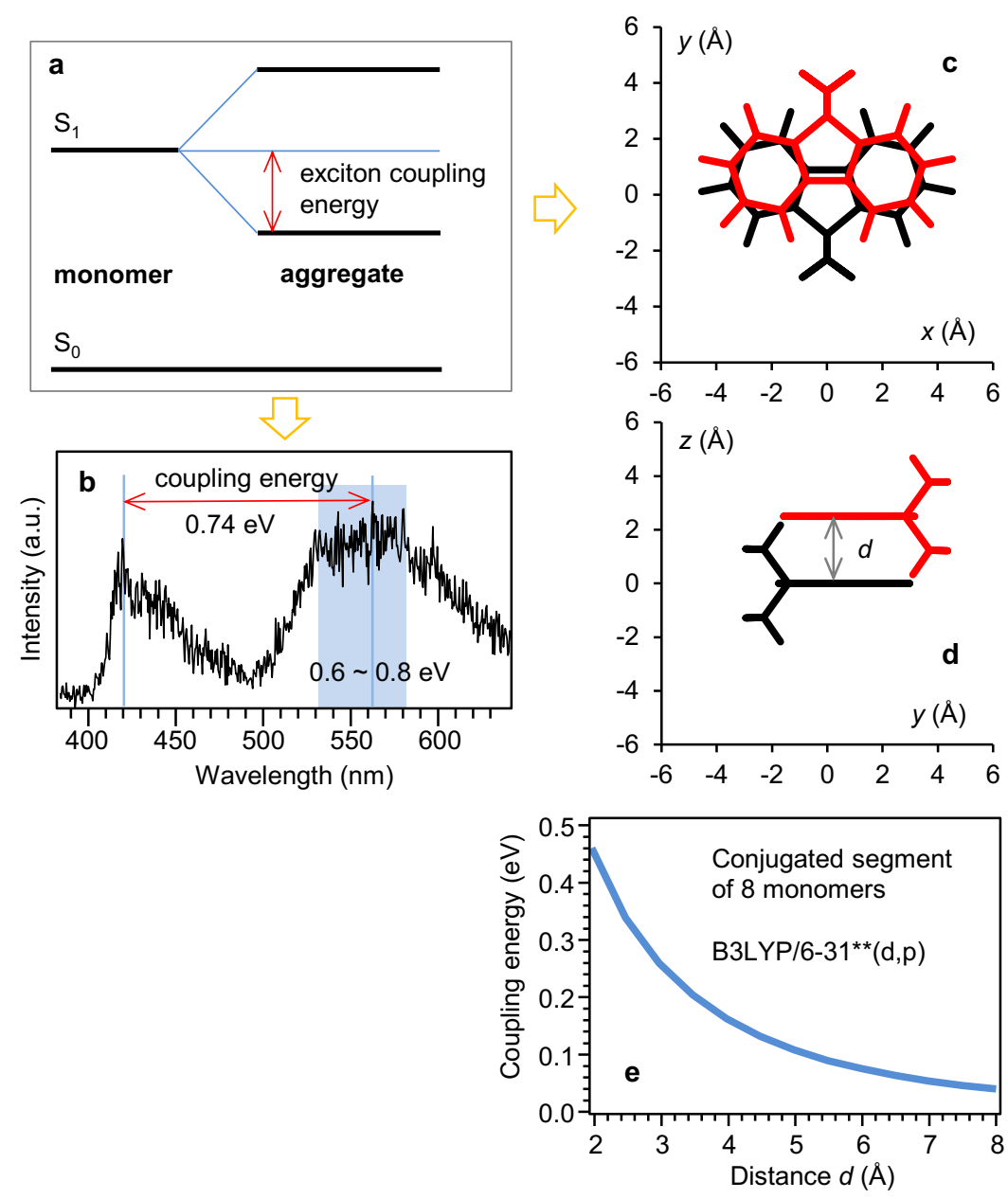

Fig. 3. Spectroscopic and quantum chemical determination of excitonic coupling. a, Schematic illustration of energy level splitting upon aggregation showing the exciton coupling energy. b, Fluorescence spectrum of a single PFO chain measured in polystyrene matrix during solvent-vapor annealing with toluene. c, d, Geometrical arrangement of face-to-face stacked fluorene dimer used in the coupling energy calculations. e, Coupling energy as a function of the distance $d$ calculated for the dimer in $\mathbf{c}, \mathbf{d}$ using the atomic transition charges method. 
The particular spectrum in Fig. $3 \mathrm{~b}$ shows contribution from both forms, and enables an estimation of the excitonic coupling energy as the difference between the maxima of the two peaks at 420.4 and $563.5 \mathrm{~nm}$, respectively. Such coupling energy is $0.74 \mathrm{eV}$, and given the broad range of the green band, the energy can vary between 0.6 and $0.8 \mathrm{eV}$. These values are in very good agreement with the $0.82 \mathrm{eV}$ energy obtained from the force spectroscopy.

Theoretically, the excitonic coupling in molecular aggregates can be separated into Coulomb interactions which are the dominant contributions at large and intermediate intermolecular distances and short-range interactions related to molecular orbitals overlap $^{34}$. For large distances the Coulombic contribution can be approximated by interaction between point transition dipoles. For distances comparable to the molecular size the contribution is more precisely calculated considering interactions between individual charges located on atoms that contain the $\pi$ electrons involved in the optical transitions $^{35}$ (atomic transition charges, ATC). Since, generally, the excitonic coupling is sufficiently well described by the Coulombic contribution ${ }^{34}$, we follow a previous approach $^{11}$ and use the ATC method to calculate the excitonic coupling energy of a faceto-face stacked fluorene dimer as a function of the intermolecular distance. The geometrical arrangement is shown in Fig. $3 c, d$, and results of the calculation for the $\mathrm{S}_{0^{-}}$ $\mathrm{S}_{1}$ transition using DFT and TD-DFT at the B3LYP/6-31G++(d,p) level are shown in Fig. 3e. The calculated coupling energy of a dimer is multiplied by 8 in the Fig. 3e to account for the average length of the PFO conjugated segment ${ }^{32}$. For a realistic range of intermolecular distances between $2 \AA$ and $8 \AA$ the calculated coupling energy ranges from $0.46 \mathrm{eV}$ to $0.023 \mathrm{eV}$. For the shorter distances in this range, these values are of the same order of magnitude as the excitonic coupling of $0.82 \mathrm{eV}$ obtained from the force 
spectroscopy. Deviations from the geometry shown in Fig. 3c, d lead to reduced coupling energy, as shown in the calculation results in Supplementary Fig. 3 and Supplementary Fig. 4. Such effects could be responsible for the deviation from linearity observed in Fig. 2f. A lack of still better agreement between the above calculated and experimental results could be caused by a non-negligible contribution of the short-range interactions or by the size of conjugated segments which could be larger that the 8 monomer units ${ }^{36}$.

\section{Conclusions and outlook}

Strong light-induced coupling between molecules in molecular aggregates resulting in an attractive force has been known for decades but direct demonstration of the coupling strength by mechanical means has not been attempted or achieved so far. We succeeded in such demonstration not only qualitatively using atomic force microscopy but to a large extent also quantitatively using single-molecule spectroscopy and quantum chemical calculations as a references. The results contribute an important piece of knowledge in the field of molecular photophysics, and will have an impact in related areas as well. In addition, the work demonstrates on molecular scale a novel energy conversion mechanism from light to mechanical energy, and this fact could have implications, e.g., in development of driving mechanisms of future molecular motors. 


\section{References}

1. Jelley, E. E. Spectral absorption and fluorescence of dyes in the molecular state. Nature 138, 1009-1010 (1936).

2. Scheibe, G., Kandler, L. \& Ecker, H., Polymerisation und polymere Adsorption als Ursache neuartiger Absorptionsbanden von organischen Farbstoffen. Naturwissenschaften 25, 75 (1937).

3. Kasha, M., Rawls H. R. \& Ashraf El-Bayoumi, M. The exciton model in molecular spectroscopy. Pure Appl. Chem. 11, 371-392 (1965).

4. Wuerthner, F., Kaiser, T. E. \& Saha-Mueller, C. R. J-aggregates: From serendipitous discovery to supramolecular engineering of functional dye materials. Angew. Chem. Int. Ed. 50, $3376-3410(2011)$.

5. J-Aggregates (Ed.: T. Kobayashi). World Scientific, Singapore, 1996.

6. Brixner, T., Hildner, R., Koehler, J., Lambert, C. \& Wuerthner, F. Exciton transport in molecular aggregates - from natural antennas to synthetic chromophore systems. Adv. Energy Mater. 7, 1700236 (2017).

7. Bialas, D., Kirchner, E., Röhr, M. I. S. \& Wuerthner, F. Perspectives in dye chemistry: a rational approach toward functional materials by understanding the aggregate state. J. Am. Chem. Soc. 143, 4500-4518 (2021).

8. Spano, F. C. \& Silva, C. H- and J-aggregate behavior in polymeric semiconductors. Annu. Rev. Phys. Chem. 65, 477-500 (2014).

9. Frenkel, J. On the transformation of light into heat in solids. Phys. Rev. 37, 17-34 (1931).

10. Davydov, A. S. Theory of Molecular Excitons. McGraw-Hill, New York, 1962. 
11. Pirrotta, A., Solomon, G. C., Franco I. \& Troisi, A. Excitonic coupling modulated by mechanical stimuli. J. Phys. Chem. Lett. 8, 4326-4332 (2017).

12. Janshoff, A., Neizert, M., Oberdorfer, Y. \& Fuchs, H. Force spectroscopy of molecular systems - single molecule spectroscopy of polymers and biomolecules. Angew. Chem. Int. Ed. 39, 3212-3237 (2000).

13. Bustamante, C., Macosko J. C. \& Wuite, G. J. L. Grabbing the cat by the tail: manipulating molecules one by one. Nat. Rev. Mol. Cell Biol. 1, 130-136 (2000).

14. Nakajima, K. \& Nishi, T. Nanoscience of single polymer chains revealed by nanofishing. Chem. Rec. 6, 249-258 (2006).

15. Giannotti, M. I. \& Vancso, G. J. Interrogation of single synthetic polymer chains and polysaccharides by afm-based force spectroscopy. ChemPhysChem 8, $2290-2307$ (2007).

16. Puchner, E. M. \& Gaub, H. E. Force and function: probing proteins with AFM-based force spectroscopy. Curr. Opin. Struct. Biol. 19, 605-614 (2009).

17. Bao, Y., Luo, Z. \& Cui, S. Environment-dependent single-chain mechanics of synthetic polymers and biomacromolecules by atomic force microscopy-based single-molecule force spectroscopy and the implications for advanced polymer materials. Chem. Soc. Rev. 49, 2799-2827 (2020).

18. Liu, Y. \& Vancso, G. J. Polymer single chain imaging, molecular forces, and nanoscale processes by atomic force microscopy: The ultimate proof of the macromolecular hypothesis. Progr. Polym. Sci. 104, 101232 (2020)

19. Hugel, T., et al. Single-molecule optomechanical cycle. Science 296, 1103-1106 (2002).

20. Sarkar, A., Robertson, R. B. \& Fernandez, J. M. Simultaneous atomic force 
microscope and fluorescence measurements of protein unfolding using a calibrated evanescent wave. Proc. Natl. Acad. Sci. USA 101, 12882-12886 (2004).

21. He, Y., Lu, M., Cao, J. \& Lu, H. P. Manipulating protein conformations by singlemolecule AFM-FRET nanoscopy. ACS Nano 6, 1221-1229 (2012).

22. Cornil, J., Beljonne, D., Calbert, J. P. \& Bredas, J. L. Interchain interactions in organic $\pi$-conjugated materials: impact on electronic structure, optical response, and charge transport. Adv. Mater. 13, 1053-1067 (2001).

23. Schwartz, B. J. Conjugated polymers as molecular materials: how chain conformation and film morphology influence energy transfer and interchain interactions. Annu. Rev. Phys. Chem. 54, 141-172 (2003).

24. Vacha, M. \& Habuchi, S. Conformation and physics of polymer chains: singlemolecule perspective. NPG Asia Mater. 2, 134-142 (2010).

25. Jenekhe, S. A. \& Osaheni, J. A. Excimers and exciplexes of conjugated polymers. Science 265, 765-768 (1994).

26. Scherf, U. \& List, E. J. Semiconducting polyfluorenes - towards reliable structureproperty relationships. Adv. Mater. 14, 477-487 (2002).

27. Knaapila, M. \& Monkman, A. P. Methods for controlling structure and photophysical properties in polyfluorene solutions and gels. Adv. Mater. 25, 1090-1108 (2013).

28. Gaal, M., List, E. J. W. \& Scherf, U. Excimers or emissive on-chain defects? Macromolecules 36, 4236-4237 (2003).

29. Honmou, Y., et al. Single molecule electroluminescence and photoluminescence of polyfluorene unveils the photophysics behind the green emission band. Nat. Commun. 5, $4666(2014)$

30. Nakamura, T., Sharma, D. K., Hirata, S. \& Vacha, M. Intra-chain aggregates as the 
origin of green emission in polyfluorene studied on ensemble and single chain level. J. Phys. Chem. C 122, 8137-8146 (2018).

31. Smith, S. B., Cui, Y. \& Bustamante, C. Overstretching b-DNA: The elastic response of individual double-stranded and single-stranded DNA molecules. Science 271, 795-799 (1996).

32. Tsoi, W. C., et al. Observation of the $\beta$-phase in two short-chain oligofluorenes. $A d v$. Funct. Mater. 18, 600-606 (2008).

33. Tseng, T. W., et al. Real-time monitoring of formation and dynamics of intra- and interchain phases in single molecules of polyfluorene. ACS Nano 14, 16096-16104 (2020),

34. Fornari, R. P., Rowe, P., Padula, D. \& Troisi, A. Importance and nature of short-range excitonic interactions in light harvesting complexes and organic Semiconductors. $J$. Chem. Theory Comput. 13, 3754-3763 (2017).

35. Chang, J. C. Monopole effects on electronic excitation interactions between large molecules. I. Application to energy transfer in chlorophylls. J. Chem. Phys. 67, 3901-3909 (1977).

36. Klaerner, G. \& Miller, R. D. Polyfluorene derivatives: effective conjugation lengths from well-defined oligomers. Macromolecules 31, 2007-2009 (1998). 


\title{
Methods
}

\section{Synthesis of the amino-terminated PFO}

\author{
1.1. Synthesis of 2-(7-bromo-9,9-dioctyl-9H-fluoren-2-yl)-4,4,5,5-tetra-methyl-1,3,2- \\ dioxaborolane
}

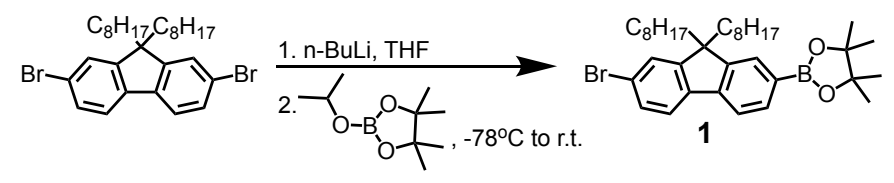

Under $\mathrm{N}_{2}$ atmosphere, 2,7-dibromo-9,9-dioctyl-9H-fluorene (2.02 g, $\left.3.7 \mathrm{mmol}\right)$ was dissolved in $20 \mathrm{~mL}$ THF and cooled to $-78^{\circ} \mathrm{C}$. BuLi $(2.4 \mathrm{~mL}, 6.0 \mathrm{mmol})$ was added dropwise to the solution and stirred for 1 hour. Then, 2-isopropoxy-4,4,5,5-tetramethyl1,3,2-dioxaborolane $(0.8 \mathrm{~mL}, 3.9 \mathrm{mmol})$ was added dropwise, the reaction mixture was heated to room temperature and kept for 24 hours. Subsequently, the organic layer was washed with saturated $\mathrm{NH}_{4} \mathrm{Cl}$ aqueous solution and dried with $\mathrm{MgSO}_{4}$. The solvent was evaporated and the crude product was purified by column chromatography using a mixed solvent of hexane $/ \mathrm{CH}_{2} \mathrm{Cl}_{2}(\mathrm{v} / \mathrm{v}=4 / 1) .0 .90 \mathrm{~g}$ colorless oil (1) was obtained as the product with yield of $41 \%$.

${ }^{1} \mathrm{H}-\mathrm{NMR}\left(\mathrm{CDCl}_{3}, 300 \mathrm{MHz}\right)$ : 7.43-7.81 (m, 6H), 1.85-2.02 (m, 4H), 1.39 (s, 12H), 0.94$1.28(\mathrm{~m}, 20 \mathrm{H}), 0.82(\mathrm{t}, \mathrm{J}=6.8 \mathrm{~Hz}, 6 \mathrm{H}), 0.49-0.62(\mathrm{~m}, 4 \mathrm{H}) . \mathrm{MS}: \mathrm{m} / \mathrm{z} 594.50$ (calc. 595.495). 


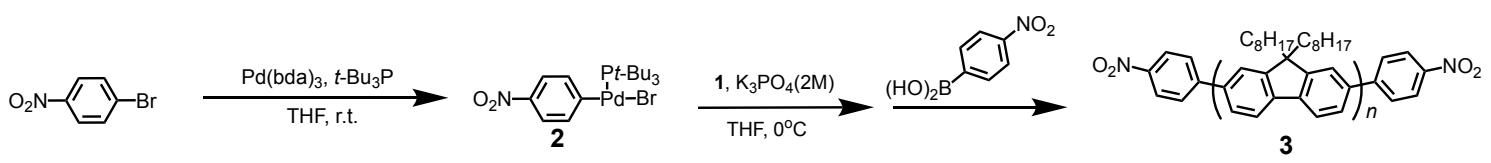

$\mathrm{Pd}(\mathrm{dba})_{2}(10 \mathrm{mg}, 0.01 \mathrm{mmol})$, tert-Bu $3 \mathrm{P}(26 \mathrm{mg}, 0.06 \mathrm{mmol})$ and 1-bromo-4-nitrobenzene (18 mg, $0.045 \mathrm{mmol}$ ) were dissolved in THF. The solution was stirred for $2 \mathrm{~h}$ in $\mathrm{N}_{2}$ and used as an initiator (2). In another flask, $1(0.28 \mathrm{~g}, 0.47 \mathrm{mmol})$ was dissolved in THF (5.4 $\mathrm{mL})$ and $2 \mathrm{M}$ aqueous solution of $\mathrm{K}_{3} \mathrm{PO}_{4}(0.5 \mathrm{~mL})$ in $\mathrm{N}_{2}$. Initiator solution was added to this mixture at $0{ }^{\circ} \mathrm{C}$ to initiate polymerization. After $1 \mathrm{~h}, 4$-nitrophenylboronic acid $(82.4$ $\mathrm{mg}, 0.49 \mathrm{mmol}$ ) was added to the reaction mixture for end capping. The product was extracted 3 times with $\mathrm{CH}_{2} \mathrm{Cl}_{2}$. Extracted organic layers were combined, washed with brine and dried with $\mathrm{Na}_{2} \mathrm{SO}_{4} . \mathrm{Na}_{2} \mathrm{SO}_{4}$ was removed by filtration and the filtrate was evaporated under reduced pressure. The residue was dissolved in a minimum amount of THF and added dropwise to methanol with stirring. The precipitate was collected by filtration $(0.1 \mathrm{~g}$, yield: $36 \%)$. The product (3) was further purified by HPLC $(0.05 \mathrm{~g}$, yield: $18 \%)$.

${ }^{1} \mathrm{H}-\mathrm{NMR}\left(\mathrm{CDCl}_{3}, 400 \mathrm{MHz}\right): 0.81$ (m, 10H), 1.14 (m, 20H), 2.12 (br, 4H), 7.68-7.70 (m, 4H), 7.83-7.85 (d, 2H). GPC: $\mathrm{M}_{\mathrm{n}}=27650, \mathrm{M}_{\mathrm{w}} / \mathrm{M}_{\mathrm{n}}=1.61$.

\subsection{Synthesis of amino-terminated $\mathrm{PFO}\left(\mathrm{NH}_{2}-\mathrm{PFO}-\mathrm{NH}_{2}\right)$}




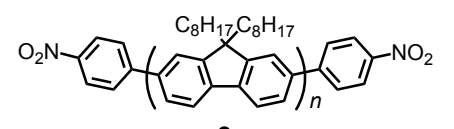

3

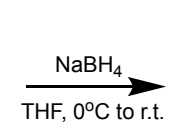

THF, oㅇ to r.t.

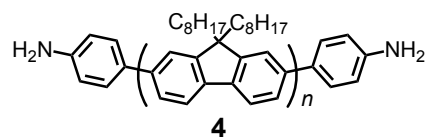

Compound 3 (20 mg, $0.7 \mu \mathrm{mol})$ was dissolved in THF and cooled to $0{ }^{\circ} \mathrm{C}$. Excessive amount of $\mathrm{NaBH}_{4}$ was added to this solution. Then, the reaction mixture was heated to room temperature and stirred for $6 \mathrm{~h}$. After filtration, organic layer was extracted with $\mathrm{CH}_{2} \mathrm{Cl}_{2}$ and dried with $\mathrm{Na}_{2} \mathrm{SO}_{4}$. The organic solvent was removed under reduced pressure and the residue was purified by the reprecipitation method with $\mathrm{CH}_{2} \mathrm{Cl}_{2} / \mathrm{methanol}(15 \mathrm{mg}$, yield: $75 \%)$.

\section{Sample preparation}

\subsection{Functionalization of quartz substrate}

First, quartz substrate was sonicated two times in ethanol for 20 min and transferred into alkaline solution $\left(\mathrm{NH}_{4} \mathrm{OH} / \mathrm{H}_{2} \mathrm{O}_{2} / \mathrm{H}_{2} \mathrm{O}, 1: 1: 5 \mathrm{v} / \mathrm{v}\right)$ for $1 \mathrm{~h}$. To obtain -OH rich surface, quartz substrate was soaked in piranha solution $\left(\mathrm{H}_{2} \mathrm{SO}_{4} / \mathrm{H}_{2} \mathrm{O}_{2}, 7: 3 \mathrm{v} / \mathrm{v}\right)$ for 30 minutes. The piranha solution was then completely removed by rinsing with water. To functionalize the surface with epoxy groups, the cleaned quartz substrate was incubated overnight in 3-glycidyloxypropyltrimethoxysilane (TCI) and thoroughly rinsed with ethanol and toluene.

\subsection{Immobilization of $\mathrm{NH}_{2}-\mathrm{PFO}-\mathrm{NH}_{2}$ on a quartz substrate}

$10^{-6} \sim 10^{-8} \mathrm{M}$ of amino terminated PFO $\left(\mathrm{NH}_{2}-\mathrm{PFO}-\mathrm{NH}_{2}\right)$ in THF solution was 
dropped onto the functionalized substrate. After THF was completely dried, the substrate was rinsed with toluene to remove the unreacted polymer chains and dried with $\mathrm{N}_{2}$ gas.

\subsection{Functionalization of AFM cantilever}

AFM cantilever was functionalized in almost the same way as the quartz substrate. First, the cantilever was immersed in ethanol for $1 \mathrm{~h}$ and transferred into alkaline solution $\left(\mathrm{NH}_{4} \mathrm{OH} / \mathrm{H}_{2} \mathrm{O}_{2} / \mathrm{H}_{2} \mathrm{O}, 1: 1: 5 \mathrm{v} / \mathrm{v}\right)$ for $1 \mathrm{~h}$. Then, the cantilever was soaked in piranha solution $\left(\mathrm{H}_{2} \mathrm{SO}_{4} / \mathrm{H}_{2} \mathrm{O}_{2}, 7: 3 \mathrm{v} / \mathrm{v}\right)$ for 30 minutes and thoroughly rinsed with water. Finally, the cleaned cantilever was incubated overnight in 3-Glycidyloxypropyltrimethoxysilane (TCI) and thoroughly rinsed with ethanol and toluene.

\section{Combined confocal and atomic force microscopic setup}

The single molecule and AFM measurements were carried out using a home-built setup that combines an inverted fluorescence microscope (IX 73, Olympus) with an AFM head (MFP-3D-SA, Asylum Research). The AFM cantilever used was HQ:CSC38/Al BS (MikroMasch). Nanofishing experiments were performed in toluene. The sample was excited with a $375 \mathrm{~nm}$ laser (LDH-D-C375, Pico Quant). Fluorescence from the sample was collected by an oil immersion objective lens (UplanFLN 100×, N.A. 1.3, Olympus) and passed through a dichroic mirror (Dichro 375, Chroma) and a long-pass filter (LP 377, Edmund). The objective lens was mounted on a three-axis piezo stage (PS3L60030U, NC3311, Nanocontrol) to accurately align its center position with respect to the AFM tip. The fluorescence signal was detected by an electron-multiplying charge- 
coupled device (EMCCD) camera (iXon, Andor Technology) equipped with an imaging spectrograph (CLP-50LD, Bunkou Keiki) to measure fluorescence spectra. The integration time and gain of EMCCD were $0.2 \mathrm{~s}$ and 300, respectively.

\section{Theoretical calculation of excitonic coupling between two PFO segments}

Excitonic coupling $J$ is calculated following the same way as reported recently for simulation of force spectroscopy of PDI molecule dimer ${ }^{11}$. The Coulombic interaction is calculated as the interaction between atomic transition charges (ATCs). ATCs for $p$-th atom in the $k$-th excited state was expressed as

$$
q_{p}^{k}=\sqrt{2} \sum_{b}^{N_{A O, p}} \sum_{c}^{N_{A O}} \sum_{j}^{\text {unocc occ }} \sum_{i}^{\text {occ }} A_{i j} C_{i}^{p b} C_{j}^{c} S_{b c}
$$

where $i$ is the index of occupied molecular orbital (MO), $j$ is the index of virtual unoccupied MOs, $b$ and $c$ are indexes of atomic orbital basis function, $N_{A O}$ is the total number of atomic orbitals, $N_{A O, p}$ is the total number of atomic orbitals of $p$-th atom, $A_{i j}$ is the CI coefficient, $C_{i}^{p b}$ is the atomic orbital coefficient $b$ of molecular orbital $i$ of $p$-th atom, $C_{j}^{c}$ is the atomic orbital coefficient $b$ of $\mathrm{MO} j$ and $S_{b c}$ is the overlap matrix between the AO basis functions $b$ and $c$. The excitonic coupling $J_{\mathrm{c}}$ was calculated using the equation (4-2) 


$$
J_{\mathrm{c}}=J_{\mathrm{c}}^{I J}=\sum_{I J} \frac{q_{i} q_{j}}{4 \pi \varepsilon_{O} r_{i j}}
$$

where $r_{i j}$ is the distance between atom $i$ and $j$ of molecule $I$ and $J$ and $\varepsilon_{0}$ is the vacuum permittivity. The ATCs of each atom were calculated from isolated fluorene monomer for $\mathrm{S}_{0}-\mathrm{S}_{1}$ transition using DFT and TD-DFT at B3LYP / 6-31G++(d,p) level. Because the

effective conjugation length of PFO is 8 monomer units ${ }^{32}$, the calculated $J_{\mathrm{c}}$ between the two fluorenes was multiplied by the factor of 8 .

\section{Data availability}

Data available on request from the authors.

\section{Acknowledgments}

The research was financially supported by the JSPS KAKENHI grants number 19H02684 and number $21 \mathrm{~K} 18927$. 


\section{Author contributions}

M.V. and K.N. conceived the idea, designed the research and discussed the results. T.N. synthesized the polymer and performed the optical and AFM experiments. X.L. assisted in the AFM experiments and discussed the results. S.O. and T.N. carried out the quantum chemical calculations. T.N. analyzed the results. T.N. and M.V. wrote the paper.

\section{Competing interest}

The authors declare no competing interests.

\section{Additional information}

Supplementary information The online version contains supplementary material available at Correspondence and requests for materials should be addressed to MV. Reprints and permissions information is available at www.nature.com/reprints. 


\section{Supplementary Files}

This is a list of supplementary files associated with this preprint. Click to download.

- NakamuraetalSI.pdf 